\title{
A Case Report of Carbohydrate Antigen 19-9 Producing Advanced Gastric Cancer
}

\author{
Konomi Mizuguchi ${ }^{1}$, Koichi Sato ${ }^{1}$, Hiroshi Maekawa ${ }^{1}$, Mutsumi Sakurada ${ }^{1}$, Hajime Orita ${ }^{1}$, Tomoyuki Kushida ${ }^{1}$, \\ Kouji Senuma ${ }^{1}$, Tomoaki Ito ${ }^{1}$, Hirokazu Matsuzawa ${ }^{1}$, Syunsuke Watanabe ${ }^{1}$, Satoshi Tokuda ${ }^{1}$, Syuhei Ueda $^{1}$ \& \\ Ryo Wada $^{2}$ \\ ${ }^{1}$ Department of Surgery, Shizuoka Hospital, Juntendo University School of Medicine, Japan \\ ${ }^{2}$ Department of Pathology, Shizuoka Hospital, Juntendo University School of Medicine, Japan \\ Correspondence: Hajime Orita, Department of Surgery, Shizuoka Hospital, Juntendo University School of \\ Medicine, Japan. E-mail: oriori@juntendo.ac.jp
}

Received: January 13, 2016 Accepted: February 2, 2016 Online Published: October 27, 2016

doi:10.5539/cco.v5n2p49 URL: http://dx.doi.org/10.5539/cco.v5n2p49

\begin{abstract}
The gastric cancer producing carbohydrate antigen 19-9 (CA 19-9) is a rare and unknown that characterize informations.

74-year-old woman who was admitted with complaints of epigastric discomfort. An advanced cancer was found in her lower gastric region; biopsy of the tumor revealed poorly-differentiated adenocarcinoma. Her serum CA 19-9 was extremely elevated at $2322 \mathrm{U} / \mathrm{ml}$ and computed tomography demonstrated enlargement of the para-aortic lymph node; thus the tumor was considered unresectable. The patient received 8 cycles of chemotherapy with S-1/cisplatin, which shrank the para-aortic lymph node dramatically, hence she underwent D2 gastrectomy. Immunohistochemical staining of the resected cancer revealed that a third of the cancer cells were positive for CA 19-9. In addition, her serum CA 19-9 decreased rapidly after surgery and she remains alive without recurrence three years after surgery.
\end{abstract}

We report one high level of CA 19-9 gastric cancer case with dramatically chemosensitive.

Keywords: CA 19-9, CA 19-9 producing gastric cancer, para-aortic lymph node metastasis

\section{Introduction}

The carbohydrate antigen 19-9 (CA 19-9) is useful for detecting pancreatic and hepatobiliary cancers, as well as digestive tract malignancies. Increased serum levels of CA 19-9 are also recognized in approximately $30 \%$ of gastric cancers. CA 19-9 producing gastric cancer is comparatively rare and its clinicopathological features have yet to be fully elucidated. Therefore, there is no standard regimen for the treatment of CA 19-9 producing advanced gastric cancer. Here we report a case of CA 19-9 producing advanced gastric cancer with para-aortic lymph node metastases that became resectable after $\mathrm{S}-1 /$ cisplatin chemotherapy.

\section{Case Presentation}

A 74-year-old female who had hypertension and hyperlipidemia was admitted to another hospital with a chief complaint of epigastric discomfort. After an upper gastrointestinal endoscopy was performed, she was diagnosed with gastric cancer and referred to our hospital. On abdominal examination, no tenderness and no abdominal mass was present. The laboratory findings showed low values for red blood cell count $\left(3.1 \times 10^{6} / \mathrm{mm}^{3}\right)$, hemoglobin $(8.5 \mathrm{~g} / \mathrm{dL})$ and hematocrit $(26.2 \%)$. The serum level of CA 19-9 was substantially elevated (2322 $\mathrm{U} / \mathrm{mL}$ ) and the carcinoembryonic antigen (CEA) level was also elevated (36 ng/ml) (Table 1). An upper gastrointestinal endoscopy performed in our hospital showed a type 2 tumor measuring approximately $70 \mathrm{~mm}$ in the anterior wall of the lower gastric body (Figure 1). It was diagnosed as a poorly-differentiated adenocarcinoma by biopsy. Computed tomography demonstrated thickened gastric wall, and enlargement of para-aortic lymph node, which measured $20 \mathrm{~mm}$ in diameter (\#16a2; defined by Japanese Classification of Gastric Carcinoma $14^{\text {th }}$ edition) (Figure 2). Since the clinical stage was determined to be T3(SS)N2M1(LYM), Stage IV, and evaluated unresectable, we chose to do chemotherapy. 


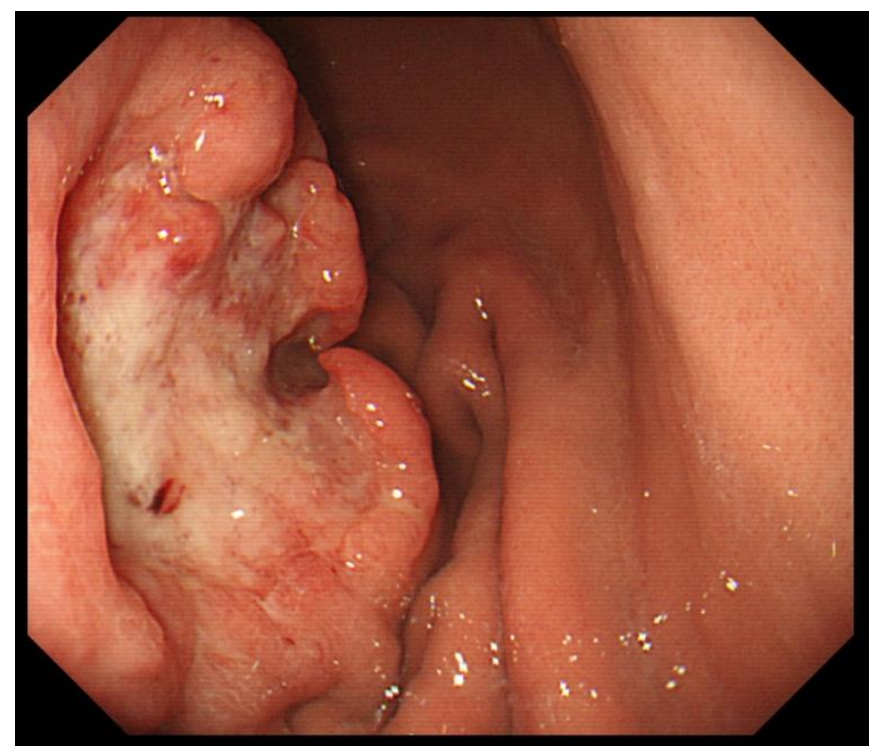

Figure 1. An upper gastrointestinal endoscopy shows a type 2 tumor measuring approximately $70 \mathrm{~mm}$ in the anterior wall of the lower gastric body

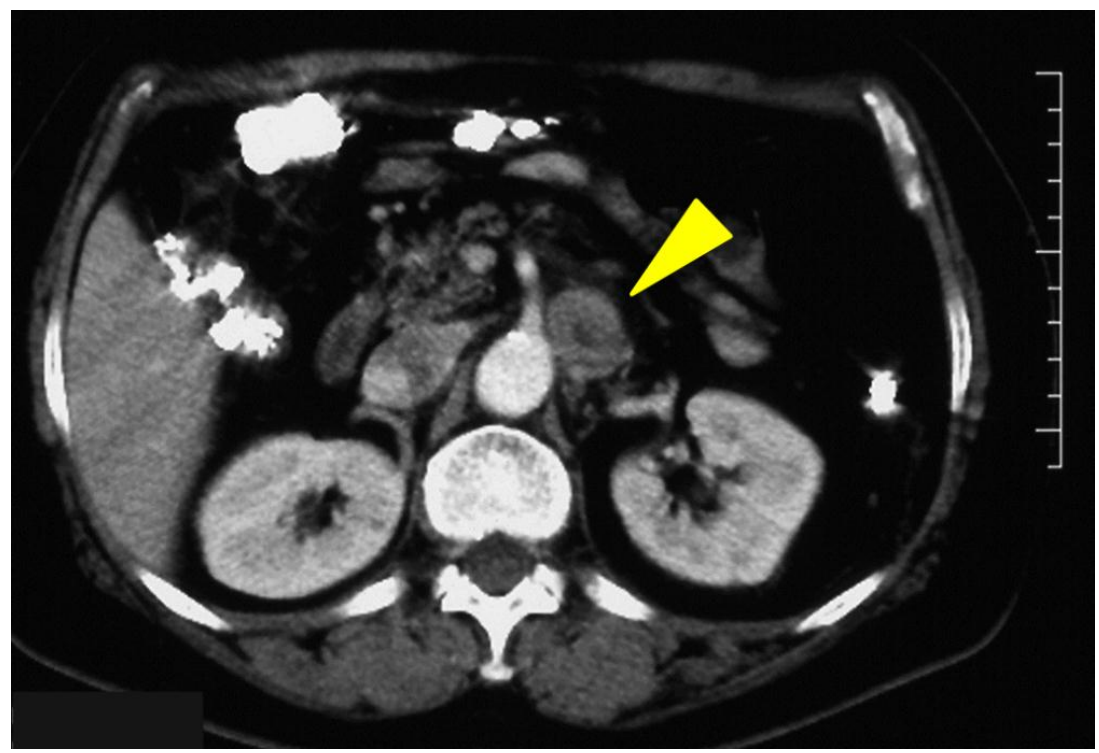

Figure 2. Computed tomography demonstrated enlargement of para-aortic lymph node, which measured $20 \mathrm{~mm}$ in diameter (\#16a2)

Therefore, the patient received chemotherapy with S-1/cisplatin. A 3-week regimen of fluoropyrimidine S-1 (40 $\mathrm{mg} / \mathrm{m}^{2}$, orally, twice daily), together with cisplatin $\left(60 \mathrm{mg} / \mathrm{m}^{2}\right.$, intravenously, day 8$)$, was administered to the patient, followed by a 2 -week rest period. The dose of chemotherapy was decreased in the second cycle, because of grade 3 nausea which was assessed according to the Common Terminology Criteria for Adverse Events version 4.0 (CTCAE v. 4.0). After the eighth cycle of chemotherapy, lymph node \#16a2 was shrunk significantly, although the primary lesion was slightly accreted (Figure 3). Levels of the CA 19-9 serum tumor marker decreased to $1090 \mathrm{U} / \mathrm{ml}$. Following eight cycles of chemotherapy, the patient underwent D2 gastrectomy and esophagojejunal Roux-en-Y anastomosis. Regarding lymph node \#16a2, the intraoperative sample was frozen and diagnosed as negative for cancer cells. 


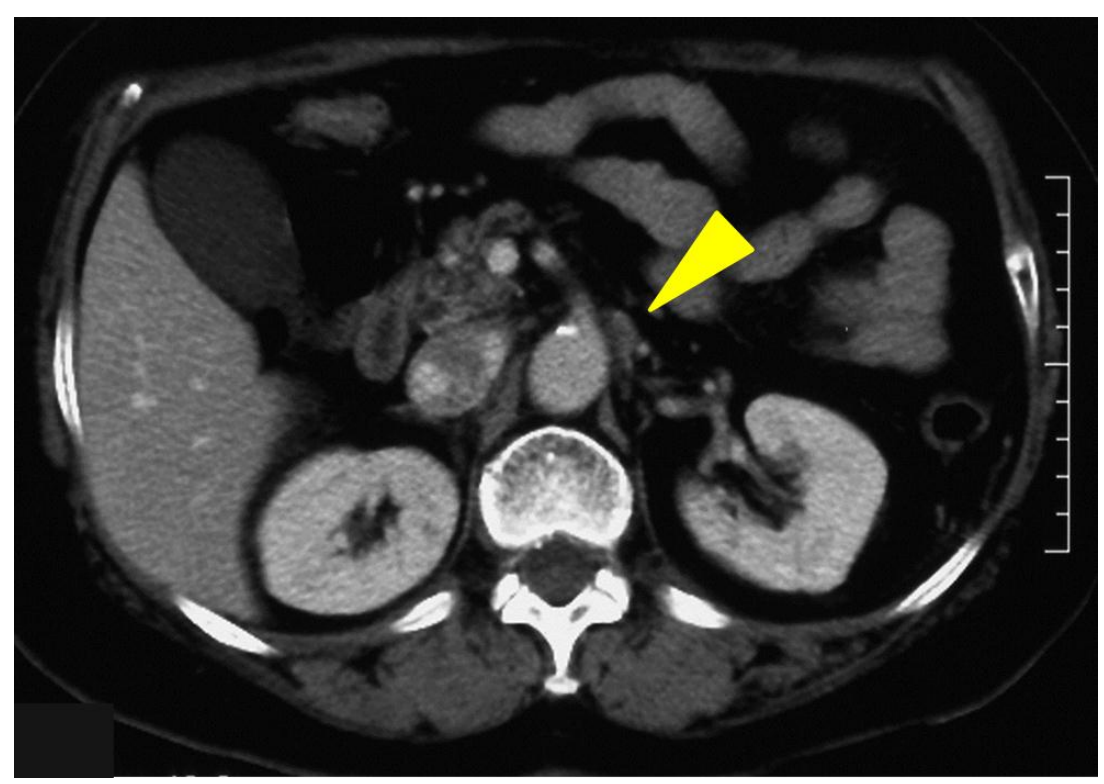

Figure 3. Lymph node \#16a2 was shrunk significantly after $8^{\text {th }}$ cycle of S-1/cisplatin chemotherapy

She had an uncomplicated postoperative course and was discharged 17 days after surgery. The serum CA 19-9 and CEA level became negative after surgery. The patient received oral fluoropyrimidine S-1 adjuvant chemotherapy for six months. She has been living for three years without recurrence.

\section{Pathological Findings}

A type 3 tumor, measuring $80 \times 60 \mathrm{~mm}$, was seen in the anterior wall of the gastric body. Microscopically, the gastric tumor was composed of poorly differentiated adenocarcinoma with directly spreading to the subserosal region, invasion to the lymphatic vessels and vein, and the metastasis in the two regional lymph nodes were found. Immunohistochemically, monoclonal anti-CA 19-9 was used at a dilution of 1:50 in the labeled streptavidin-biotin method determined on formalin-fixed, paraffin-embedded tissue. A third of cancer cells were positive for CA 19-9 (Dako Cytomation Co.) (Figure 4). The histological therapy evaluation was grade 1 according to the Japanese Classification of Gastric Carcinoma (the 14-th edition). And pathological - TNM classification of this tumor showed stage IIb (T3N1M0).

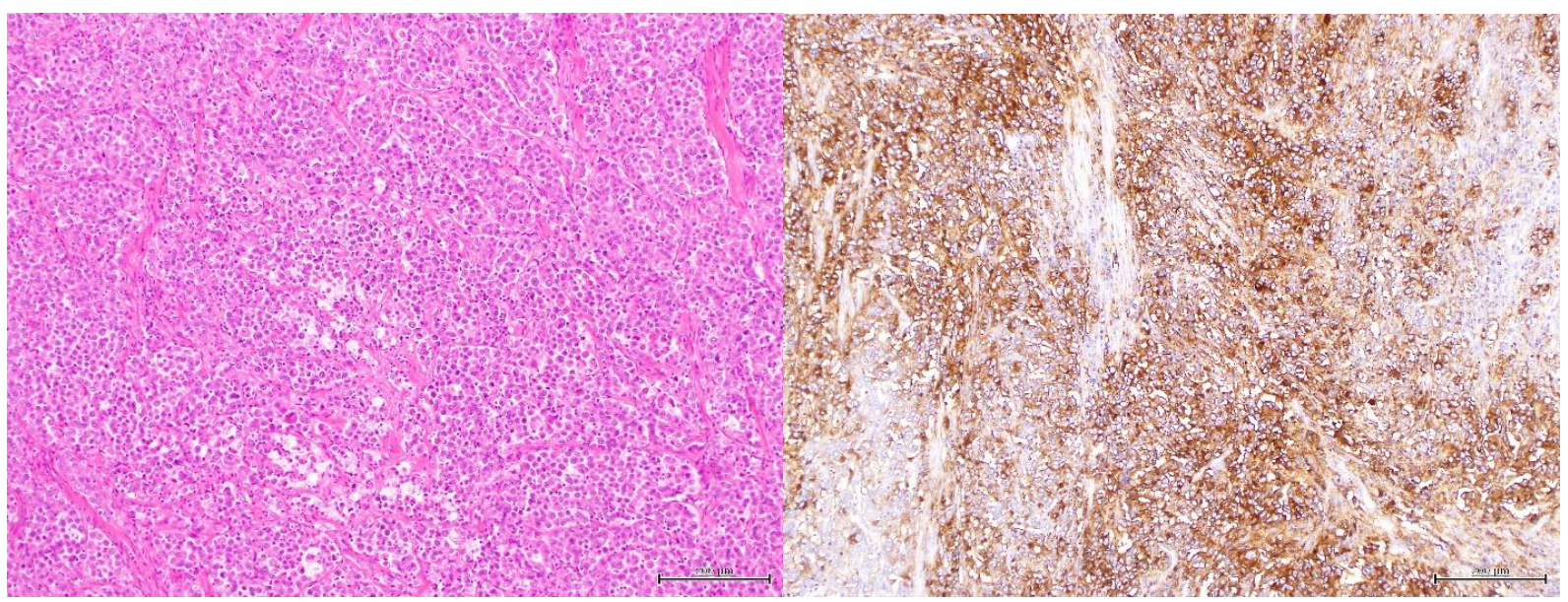

Figure 4. Immunohistological examination showed CA 19-9 staining on a third of cancer cells

\section{Discussion}

Monoclonal antibody 19-9, derived from mice spleen cells immunized with human colon adenocarcinoma cell line SW1116, can be detected in the serum of patients (Koprowski, Steplewski, Mitchell, Herlyn, Herlyn, \& Fuhrer, 1979). CA 19-9 is an effective tumor marker, as useful as CEA in detecting digestive tract malignancies. 
The tumor marker CA 19-9 is a sensitive marker for patients with pancreatic and hepatobiliary cancer, as well as gastrointestinal and colorectal cancers. Serum CA 19-9 levels are used as a means of therapy evaluation, and also to assess prognosis and postoperative recurrence.

CA 19-9 producing gastric cancer has been defined by three factors: high serum levels of CA19-9 before resection, CA19-9 antibody in resected specimens, and lower serum CA19-9 levels after resection (Okinaga, Yokohata, \& Jibu, 1994). It is relatively rare, and its clinicopathological features have yet to be fully elucidated. Since the role of CA 19-9 in gastric cancer is unclear, it is uncertain whether CA 19-9 production indicates malignant potential or not. The serum level of CA 19-9 rises in the postoperative state of recurrent cases wherein it produces gastric cancer. Although preoperative serum levels of CA 19-9 are uncorrelated with stage of cancer progression, histological type or prognosis, it is reported that serum levels of CA 19-9 are very helpful as a diagnostic tool for postoperative recurrence of it producing gastric cancers. Moreover, very high levels often indicate advanced cancer which has metastasized to the lymph nodes and liver (Hibi, Serizawa, Kyo, Takagi, Aoki, \& Mukai, 2008).

Since cases in which CA19-9 producing gastric cancer are rare, there is no standard regimen of chemotherapy. Even though some papers have reported that oral fluoropyrimidine S-1 is effective in decreasing tumor size and preventing recurrence (Sakamoto et al., 2005; Yamazaki et al., 2009; Matsutani et al., 2012). The prognosis of CA 19-9 producing gastric cancers remains poor; thus, further studies of multiagent regimens are needed. On account of the results of this case being so promising it would be beneficial to compile future cases of CA 19-9 producing cancer in order to better understand the connection.

Most gastric cancers are diagnosed at advanced stage because of silent undetected symptoms. Therefore palliative treatment is needed for these patients. A large number of papers have been published studying the efficacy of palliative chemotherapy, but a standard regimen has yet to be determined. In much of Europe, ECF (epirubicin $50 \mathrm{mg} / \mathrm{m}^{2}$ day 1 , cisplatin $60 \mathrm{mg} / \mathrm{m}^{2}$ day 1 and continuous infusion of 5-fluorouracil $200 \mathrm{mg} / \mathrm{m}^{2}$ daily) has been regarded as a reference regimen in patients with advanced gastric cancer derived from the MAGIC trial (Cunningham et al., 2006). REAL-2 trial demonstrated equivalent clinical efficacy when capecitabine was substituted for 5-fluorouracil in the ECF regimen (Cunningham et al., 2008). In many Asian countries, including Japan, S-1 based regimens are the standard treatment based on data from two randomized phase III trials in patients with metastatic gastric cancer (JCOG9912 (Boku et al., 2009), SPIRITS (Koizumi et al., 2008)). Gastric cancer with para-aortic lymph node metastasis, is classified as stage IV cancer and is generally considered non-resectable. However, some reports show a 5-year survival rate of approximately $10 \%$ after para-aortic lymph node dissection for gastric cancer with para-aortic lymph node metastasis (Ohashi et al., 1976; Tokunaga, Ohyama, Hiki, Fukunaga, Aikou, \& Yamaguchi, 2010). Furthermore, it is reported that S-1/cisplatin followed by surgery including para-aortic lymph node dissection is safe and effective for some patients (Tsuburaya, Mizusawa, Tanaka, Fukushima, Nashimoto, \& Sasako, 2014).

Our patient's, gastric cancer with para-aortic lymph node metastasis was considered non-resectable. The first line chemotherapy, S-1/cisplatin, was administered as a palliative therapy. This shrunk the para-aortic lymph dramatically, which made performing a D2 gastrectomy possible after the $8^{\text {th }}$ cycle of chemotherapy. Presently, the patient has been living for three years without recurrence and her serum level of CA 19-9 has decreased considerably.

\section{Conclusion}

Here we report a case of CA 19-9 producing advanced gastric cancer with para-aortic lymph node metastasis that became resectable after S-1/cisplatin chemotherapy. The optimal chemotherapy regimen for CA 19-9 producing gastric cancer is unknown at this time. By any chance, CA 19-9 producing gastric cancer have the potential of being used effectively in the treatment of S-1/cisplatin chemotherapy. Further studies are needed to improve the prognosis of patients with CA 19-9 advanced gastric cancer.

\section{References}

Boku, N., Yamamoto, S., Fukuda, H., Shirao, K., Doi, T., Sawaki, A., ... Nasu, J. (2009). Gastrointestinal Oncology Study Group of the Japan Clinical Oncology Group Fluorouracil versus combination of irinotecan plus cisplatin versus S-1 in metastatic gastric cancer: a randomised phase 3 study. Lancet Oncol, 10(11), 1063-1069. http://dx.doi.org/10.1016/S1470-2045(09)70259-1

Cunningham, D., Allum, W. H., Stenning, S. P., Thompson, J. N., Van de Velde, C. J., Nicolson, M., ... Smith, D. B. (2006). Perioperative chemotherapy versus surgery alone for resectable gastroesophageal cancer. New England Journal of Medicine, 355(1), 11-20. http://dx.doi.org/10.1056/NEJMoa055531 
Cunningham, D., Starling, N., Rao, S., Iveson, T., Nicolson, M., Coxon, F., ... \& Norman, A. R. (2008). Capecitabine and oxaliplatin for advanced esophagogastric cancer. New England Journal of Medicine, 358(1), 36-46. http://dx.doi.org/10.1056/NEJMoa073149

Hibi, Y., Serizawa, H., Kyo, B., \& Takagi, Y. (2008.). Aoki T and Mukai K: Clinicopathological study of CA 19-9 producing gastric cancers. Geka 70, 1524-1531.

Koizumi, W., Narahara, H., Hara, T., Takagane, A., Akiya, T., Takagi, M., ... Toh, Y. (2008). S-1 plus cisplatin versus S-1 alone for first-line treatment of advanced gastric cancer (SPIRITS trial): a phase III trial. The lancet oncology, 9(3), 215-221. http://dx.doi.org/10.1016/S1470-2045(08)70035-4

Koprowski, H., Steplewski, Z., Mitchell, K., Herlyn, M., Herlyn, D., \& Fuhrer, P. (1979). Colorectal carcinoma antigens detected by hybridoma antibodies. Somatic cell genetics, 5(6), 957-971. http://dx.doi.org/10.1007/BF01542654

Matsutani, T., Yoshida, H., Sasajima, K., Maruyama, H., Yokoyama, T., Suzuki, S., ... \& Uchida, E. (2012). [A successful resected case of far-advanced CA19-9-producing gastric cancer by neoadjuvant chemotherapy with S-1 and Cisplatin]. Gan to kagaku ryoho. Cancer \& chemotherapy, 39(4), 653-656.

Ohashi, I., Takagi, K., Konishi, T., Izumoi, S., Fukami, A., Kajitani, T., et al. (1976). Five Year Survival Cases with Dissection of Para-aortic Lymph-Node Metastases for Gastric Cancer. Jpn J Gastroenterol Surg., 9, 112-116. http://dx.doi.org/10.5833/jjgs.9.112

Okinaga, K., Yokohata, T., \& Jibu, T. (1994). CA 19-9 producing gastric cancer. Bessatsu Nihon Rinsyou, 5 , 238-240.

Sakamoto, I., Makita, F., Kashiwabara, K., Yoshimura, S., Higashi, M., Takeyoshi, I., et al. (2005). CA 19-9 Producing Gastric Cancer with a Macroscopically CR to TS-1. Jpn J Gastroenterol Surg, 38, 135-140. http://dx.doi.org/10.5833/jjgs.38.135

Tokunaga, M., Ohyama, S., Hiki, N., Fukunaga, T., Aikou, S., \& Yamaguchi, T. (2010). Can Superextended Lymph Node Dissection be Justified for Gastric Cancer with Pathologically Positive Para-aortic Lymph Nodes? Ann Surg Oncol., 17, 2031-2036. http://dx.doi.org/10.1245/s10434-010-0969-4

Tsuburaya, A., Mizusawa, J., Tanaka, Y., Fukushima, N., Nashimoto, A., \& Sasako, M. (2014). Neoadjuvant chemotherapy with S-1 and cisplatin followed by D2 gastrectomy with para-aortic lymph node dissection for gastric cancer with extensive lymph node metastasis. Br J Surg., 101, 653-660. http://dx.doi.org/10.1002/bjs.9484

Yamazaki, K., Watanabe, M., Otsuka, Y., Tamura, A., Tsuchiya, M., Yajima, S., et al. (2009). Carbohydrate Antigen 19-9 Producing Gastric Cancer: A Report of four Cases. J Med Soc Toho., 56, 219-226.

\section{Copyrights}

Copyright for this article is retained by the author(s), with first publication rights granted to the journal.

This is an open-access article distributed under the terms and conditions of the Creative Commons Attribution license (http://creativecommons.org/licenses/by/4.0/). 